\title{
CARACTERÍSTICAS VOCACIONALES DE ESTUDIANTES UNIVERSITARIOS CIEGOS Y DEFICIENTES VISUALES: UNA APROXIMACIÓN CUALITATIVA
}

\author{
VOCATIONAL CHARACTERISTICS OF UNIVERSITY BLIND \\ STUDENTS AND STUDENTS WITH VISUAL IMPAIRMENT: \\ A QUALITATIVE APPROACH
}

\author{
V. Ávila* y E. Rocabert** \\ Universidad de Valencia
}

\section{RESUMEN}

Son bien conocidos los condicionantes vocacionales que influyen en los estudiantes universitarios en función de características como el sexo, nivel socioeconómico o grupo vocacional al que se adscriben. Sin embargo, poco se conoce de las características vocacionales de estos estudiantes cuando están afectados por una discapacidad. La aproximación cualitativa, a través de entrevistas semiestructuradas, es la vía que permite conocer con detenimiento las necesidades y características de estos estudiantes para establecer un asesoramiento de calidad. Esta metodología nos muestra que dicho asesoramiento debe centrarse, básicamente, en tres campos: el socio-personal, el de autonomía e independencia personal, y el propiamente vocacional a través de servicios ofertados por la institución universitaria, como el llevado a cabo en la Universitat de València; pero sin restringir la actuación a este momento educativo. Este trabajo trata de realizar una primera prospección que sirva de base para establecer servicios especializados en la atención a estudiantes con discapacidad visual.

Palabras Clave: Asesoramiento Vocacional, Discapacidad, Universidad

\section{ABSTRACT}

Vocational charactrstics related to sex, socioeconomic status or vocational group influencing higher education students' vocational behaviour are well known. However, the vocational characteristics of

* Vicenta Ávila Clemente es Profesora Asociada de Escuela Universitaria del Departamento de Psicología Evolutiva y de la Educación de la Universidad de Valencia. Su línea de investigación se centra en el asesoramiento a estudiantes con discapacidad física o sensorial.

** Esperanza Rocabert Beut es Profesora Titular de Psicología de la Educación de la Universidad de Valencia. Su actividad profesional y líneas de investigación actuales se centran en la Psicología Vocacional, asignatura que imparte dentro de la Licenciatura de Psicopedagogía. 
students that suffer from some kind of dissability have been less studied.The qualitative apporach with it use of semi-structured interviews is the best way to find out the needs and unique characteristics of these students in order to deliver quality counseling services. Qualitative methodology shows us that vocational and career counseling should be based on three domains: the socio-personal domain, the personal autonomy domain, and the vocational domain. An example of counseling directed to the vocational domain could be the use of University-based counseling services, but should not be restricted to this educational level. This research attempts is a first step to develop specialized counseling services for students with some kind of visual impairment.

Key Words: Vocational Guidance, Dissabilities, University.

\section{Introducción}

El proceso de integración escolar, iniciado en nuestro país hace algunas décadas, unido a la implantación de la Ley de Ordenación General del Sistema Educativo, ha introducido nuevos elementos que condicionan el desarrollo vocacional. Así, aunque son todavía pocos los estudiantes con discapacidad que completan la enseñanza no obligatoria y acceden a la Universidad, tal y como demuestra el trabajo realizado por la Fundación ONCE (1996) sobre minusvalía e inserción laboral en la Comunidad Valenciana en el que se señala que sólo un 5,3\% de las personas con discapacidad de nuestra comunidad tiene formación universitaria; esta población ha ido emergiendo en estudios postobligatorios, entre los que parecen ser las personas con discapacidad visual quienes han iniciado este camino.

Se repite en este colectivo la situación observada respecto a la población general: el hecho de realizar estudios universitarios no garantiza la obtención de empleo, aunque influye positivamente. La precariedad en la oferta de empleo, que afecta a todos los trabajadores, repercute de manera más directa en colectivos con dificultades; existiendo una correlación positiva, también en este colectivo, entre nivel de educación y ocupación (Fundación ONCE (o.c); Bush-LaFrance, 1988).

Se hacen necesarias medidas de discriminación positiva, para favorecer el incremento de su nivel de estudios y la posibilidad de cursar estudios universitarios. Una de esas medidas pasa por el establecimiento de servicios de asesoramiento que oferten un apoyo de calidad, haciéndose necesario que los profesionales tengan un mayor conocimiento de las características y necesidades de este grupo de estudiantes, y centrando su trabajo en tres campos (Rocabert, 1999): socio-personal, autonomía e independencia personal, y vocacional. Sin olvidar la complejidad que este grupo de población representa; ya que son muchos los tipos de déficits con los que nos encontramos, e implica una gran heterogeneidad del colectivo. Todo ello hace más complicada la atención sin un conocimiento previo y en profundidad de la población de referencia. En este trabajo presentamos parte de los resultados obtenidos en la Universitat de València (Estudi General), en adelante UVEG, relativos al análisis del grupo de estudiantes con discapacidad visual (Ávila, 1998).

\section{Objetivos}

Nuestro fin último y principal se centraba en conseguir que el proceso de asesoramiento vocacional dirigido a estudiantes con discapacidad visual contenga los elementos necesarios para ofrecer un servicio de calidad, es decir que permita al estudiante por una parte pro- 
gresar en el proceso de enseñanza/aprendizaje en los estudios superiores, pero también posibilitar el que se tomen decisiones vocacionales realistas partiendo de un adecuado conocimiento de la conducta vocacional, del mundo laboral y académico.

Para realizar el análisis del desarrollo vocacional, partimos del conocimiento de la conducta vocacional como elemento que forma parte del proceso de socialización de cualquier individuo, revisando algunos de los condicionantes de este proceso, apuntados por Rivas (1995). Este autor diferencia dos tipos de condicionantes, los psicogenéticos o factores individuales (biodatos, intereses, aptitudes, etc.), que son intrínsecos al sujeto, y los sociogenéticos o factores supraindividuales que se encuentran fuera del ámbito individual del sujeto y que no son controlables por él (mercado laboral, sistema educativo, etc.).

Partimos de la asunción de que la conducta vocacional de las personas con discapacidad no es distinta de la del resto de individuos. Sus condicionantes vocacionales serán los mismos; pero estarán mediatizados por las limitaciones funcionales que el déficit conlleva, por lo tanto, al igual que lo apuntado por Fitzgeral y Crites (1980) en el caso de las mujeres, el desarrollo vocacional no es diferente sino más complejo (Rocabert, 1999).

Sin embargo, sí existe cierta especificidad en el desarrollo de carrera y en los procesos de búsqueda de empleo en este grupo de población. Rabby y Croft (1989) afirman que esa parte específica, o diferente, podría calibrarse en tan sólo un $5 \%$ de los procesos y no sería consecuencia tanto de la propia discapacidad, como de la existencia de barreras sociales y de concepciones erróneas en cuanto a las capacidades del individuo. Tratamos, pues en nuestro trabajo, conocer mejor los elementos de esa parte específica del desarrollo vocacional del grupo de estudiantes con discapacidad visual.

A su vez es también tradicional encontrar determinadas ideas preconcebidas, reales o no, en la percepción del mundo académico y laboral que influyen en el desarrollo vocacional de estas personas. Para su análisis hemos partido de los trabajos de Alcantud (1994) y Salomone y Paige (1996), en los que se ponen de relieve los aspectos que pueden suponer factores de segregación en el ámbito universitario y circunstancias que se muestran como obstáculos para la inserción socio-laboral de personas con discapacidad visual, respectivamente.

Entre los factores segregacionistas Alcantud (1994) señala cuatro: los aspectos relacionados con la aptitud física y el aprovechamiento, los relacionados con la sanidad y la seguridad, las posibles dificultades en relaciones sociales y las actitudes de la comunidad universitaria. Respecto a la inserción socio-laboral Salomone y Paige (1996) apuntan como principales barreras: la actitud negativa de la gente hacia la discapacidad, el pobre autoconcepto que pueden mostrar las personas con déficit, la negativa de los empresarios a contratar, así como el transporte limitado.

Esta revisión teórica nos llevó a contemplar para este trabajo, que se enmarca en otro más amplio, un conjunto de cinco grandes grupos de variables, que pasamos a describir mas detenidamente:

A) Variables Demográficas: dentro de este apartado nos interesaba resaltar los siguientes aspectos:

- Edad (Menores de 25 años, entre 25-40 años, o mayores de 40 años).

- Sexo (Mujeres y Hombres).

- Grado de Discapacidad (Más del 65\%; entre el 33 y el 65\%). 
- Tipo de Discapacidad (Ciegos, Deficientes Visuales con enfermedad degenerativa; Deficientes Visuales sin enfermedad degenerativa).

- Momento de Aparición del Déficit (Congénito, entre 0 y 3 años, Entre 3 y 13 años, Entre 14 y 18 años y a partir de los 18 años).

- Incidencia de la misma Discapacidad en la Familia (ninguna, en hermanos, en padres).

B) Factores Condicionantes de la Conducta Vocacional: En este apartado se analizan variables como la Familia (Status, y actitud) así como los biodatos de tipo académicos.

- Familia: Dentro de las variables familiares, el Status Sociocultural y Económico (alto, medio o bajo) se ha mostrado como uno de los mayores condicionantes de la conducta vocacional de los Hijos. A su vez y sobre todo en las familias con hijos con discapacidad, la Actitud y las Expectativas (apoyo, oposición, o indiferencia) de los padres, percibidas por el hijo/a, en relación a su rol escolar y laboral, se ha comprobado que puede tener una gran influencia en el desarrollo vocacional de estas personas.

- Biodatos Académicos: En el contexto español las experiencias y actitudes relacionadas con la formación académica (rendimiento académico, itinerario escogido, la preferencia por ciertas asignaturas) condiciona mucho la conducta vocacional de los individuos. A ello hay que añadir la influencia que para este tipo de personas tiene el hecho de haber realizado sus estudios en Centros Específicos o en Régimen de Integración, así como el momento de inicio de su escolarización.

C) Factores Condicionantes de la Elección Vocacional: Para nuestro análisis sobre qué factores influyen en la elección de unos estudios universitarios u otros hemos contemplado la influencia de los siguientes aspectos: el propio Déficit, la influencia de la Familia, Profesores, Amigos, la ONCE, Modelos a imitar, Aptitudes, Preferencias, las Salidas Profesionales, la Accesibilidad al centro de formación, así como la posibilidad de Compaginar con otras Actividades, la posibilidad de Aumentar la Cultura o el Asesoramiento Vocacional recibido.

D) Tipo de Elección Vocacional: En este apartado contemplamos tanto los estudios universitarios concretos (titulación) que se realizan; así como la salida laboral a la que aspiran, en ambos casos se tiene en cuenta la característica de tradicionalidad o no para personas con déficit visual de la elección realizada.

E) Percepción de Barreras Universitarias: Este grupo de variables analiza las dificultades o percepción de barreras que los estudiantes con déficits visuales tienen durante el transcurso de sus estudios (barreras arquitectónicas y de infraestructura; organización escolar; material académico; metodología; y actitud del profesorado y de los compañeros).

F) Percepción de Barreras Laborales: Recoge la opinión de la muestra en relación a posibles barreras a la hora de encontrar y desarrollar un trabajo: Actitud de la sociedad hacia la discapacidad visual; autoconcepto, actitud de los empresarios a la hora del contrato; limitaciones en el transporte; y preparación vocacional recibida. 


\section{Metodología e Instrumentación}

A través de la Asesoría Universitaria de Estudiantes con Discapacidad de la UVEG, contactamos telefónicamente con los estudiantes universitarios que durante el curso 1997/98, se autodeclararon como personas con discapacidad visual.

Con el fin de cubrir el objetivo de conocer en mayor profundidad la conducta vocacional y la percepción del mundo académico y laboral de estos sujetos, se elaboró un cuestionario guía (ver anexo I), que contempla las variables anteriormente mencionadas.

Este cuestionario se implementó a través de entrevistas semiestructuradas mantenidas con los sujetos de la muestra en los locales de la Asesoría.

En el momento de realizar nuestro trabajo, contábamos con un total de 33 estudiantes con discapacidad visual, según el censo proporcionado por la Asesoría Universitaria de Atención a Estudiantes con Discapacidad, que cursaban estudios en la UVEG, que suponía el 23,4\% del total de estudiantes con discapacidad. De este número, un total de 14 personas accedieron a participar en la investigación. Debido al pequeño número al que nos enfrentamos, optamos por realizar entrevistas semiestructuradas, basadas en el cuestionario guía elaborado para tal fin, en las que se intentó perfilar la historia de vida de los individuos, tomando como referentes los trabajos llevados a cabo por Hurst (1993) y por Hutto (1993). Se trata de trabajos que aglutinan tanto la tradición cuantitativa como cualitativa. Denzin y Lincoln (1994) lo denominan «triangulación» (triangulation), apoyando la utilización de métodos cuantitativos y cualitativos en una misma investigación, a fin de obtener los mejores resultados posibles.

Dada las características de la muestra y la información recogida, los análisis realizados se limitan a una descripción cualitativa basada en porcentajes y a los comentarios obtenidos en las entrevistas.

\section{Resultados}

A continuación pasamos a reflejar los principales resultados obtenidos tras el análisis de porcentajes de las respuestas dadas al cuestionario, así como de los comentarios más relevantes surgidos en la entrevista mantenida con los estudiantes:

\section{A) Variables Demográficas}

En la tabla 1 queda reflejada la distribución de los sujetos en función de las variables y categorías establecidas para este apartado.

\section{B) Factores Condicionantes de la Conducta Vocacional}

\section{Familia}

La familia es el primer referente de cualquier individuo y como tal influirá en la conducta vocacional de todos sus miembros. Tradicionalmente, el nivel sociocultural y econó- 
TABLA 1.

\begin{tabular}{|l|l|r|}
\hline \multirow{2}{*}{ Edad } & Menores de 25 años & $46,6 \%$ \\
& Entre 25 y 40 años & $40 \%$ \\
& Mayores de 40 & $13,3 \%$ \\
\hline \multirow{2}{*}{ Sexo } & Mujeres: & $53 \%$ \\
& Hombres: & $47 \%$ \\
\hline \multirow{2}{*}{ Grado de discapacidad } & Más del 65\%: & $86 \%$ \\
& Entre 33 y 65\%: & $14 \%$ \\
\hline \multirow{5}{*}{ Tipo de Discapacidad } & Ciegos: & $40 \%$ \\
& Def. Visuales (enfermedad degenerativa): & $20 \%$ \\
& Def. Visuales (enfermedad no degenerativa): & $40 \%$ \\
\hline \multirow{3}{*}{ Momento de Aparición } & Congénito (0-3 años): & $60 \%$ \\
& Adq. Infancia (3-13 años): & $20 \%$ \\
& Adq. Adolescencia (14-18 años): & $13 \%$ \\
& Adq. Juventud (+18 años): & $7 \%$ \\
\hline \multirow{2}{*}{ Incidencia de la } & Ninguna: & $69 \%$ \\
& Hermanos: & $23 \%$ \\
& Madre: & $8 \%$ \\
\hline
\end{tabular}

mico de la familia de origen era un factor no sólo condicionante, sino determinante en la realización de estudios universitarios. En nuestro entorno dadas las características de la Universidad actual, donde existen programas de becas dirigidos a paliar las barreras socioeconómicas de los estudiantes, este factor ha dejado de ser determinante aunque sigue siendo condicionante según muchos autores; $\mathrm{y}$ especialmente en personas con algún tipo de déficit (Hutto, 1993; Alcantud, 1995).

Si el nivel sociocultural de la familia de origen lo definimos en función del nivel de estudios de los padres y de su ocupación laboral, contradiciendo los trabajos anteriores, en nuestra experiencia más del $60 \%$ de las familias las podríamos enmarcar en un nivel mediobajo, caracterizado por estudios primarios y una ocupación que se corresponde con dicho nivel de estudios (Figs. 1 y 2).

Este hecho puede deberse al papel compensatorio de las becas de estudio que la ONCE oferta a estos estudiantes. Hay que subrayar que un $40 \%$ de los entrevistados disponen de ellas.

Uno de los principales influjos que la familia ejerce, viene de la actitud ante el rol escolar y laboral de los hijos y de las expectativas laborales que se tengan sobre ellos. En el caso de hijos con déficits las actitudes de sobreprotección, falta de expectativas o de negación del problema; todas ellas impropias, se reflejarán en la conducta general del individuo $y$, por lo tanto, también en su conducta vocacional.

Encontramos que las actitudes que las familias han mostrado han provocado situaciones bien distintas: personas que han reaccionado contra la sobreprotección, sobre todo por parte de sus madres, tratando de alejarse de la familia y buscando la independencia que se les negaba y personas que siendo manifiesta la sobreprotección de la familia, la aceptan e incluso se aprovechan de ella. 


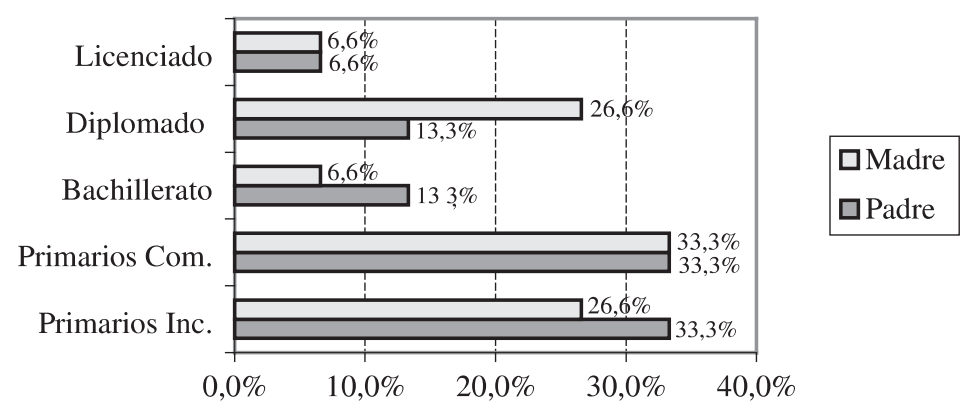

FIGURA 1.

Nivel de estudio de los padres

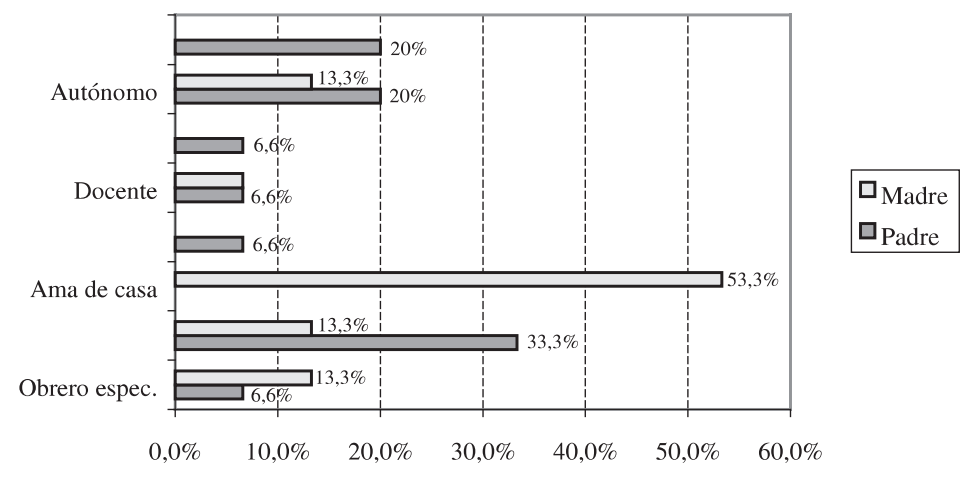

FIGURA 2.

Ocupación de los padres.

\section{Biodatos Académicos}

La historia académica de los individuos condiciona gran parte de su conducta vocacional, máxime si tenemos en cuenta que la escolarización se inicia a edades muy tempranas. Entre los entrevistados el 20\% inició su escolarización antes de los 4 años, el 66,6\% lo hicieron entre los 4 y los 6 años y tan sólo un 13,3\% comenzaron su escolaridad con posterioridad a los 6 años.

En lo referente a los datos académicos, la escolarización de personas con discapacidad visual ha estado durante más de tres décadas, por delegación del Estado, única y exclusivamente en manos de la ONCE. Esta situación comenzó a cambiar hace aproximadamente diez años tras la promulgación de la LOGSE. Las edades de los estudiantes universitarios constataban que un alto porcentaje, el 53,3\%, estuvieron escolarizados en centros específicos para ciegos. El 46,6\% restante son personas que adquirieron el déficit en etapas poste- 
riores de su ciclo vital o aquellas que, sufriendo una discapacidad visual, no se les consideraba ciegos (Fig. 3).

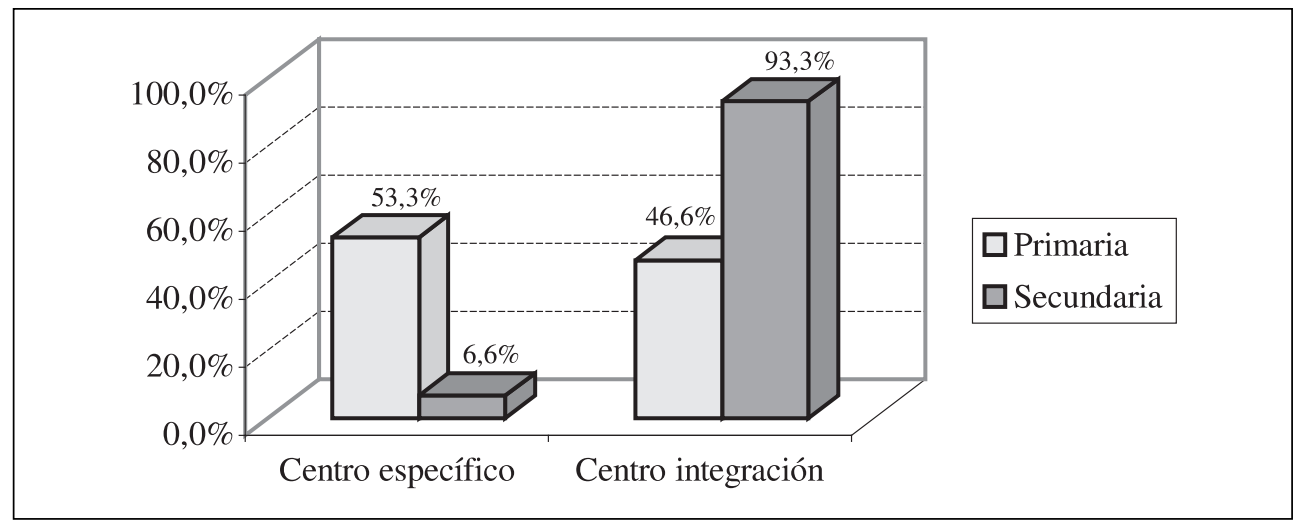

FIGURA 3.

Tipo de Centro en que se realizó la enseñanza no universitaria.

Con la llegada de la integración escolar, la mayoría de personas pasaron, ya en primaria, a centros integrados y ninguno, a excepción de una persona con más de 40 años que recibió también formación profesional en centro específico, realizaron estudios secundarios en régimen de integración.

El 14\% de las personas entrevistadas no cursó BUP (realizaron el acceso a la Universidad mediante la prueba de mayores de 25 años), de las personas que sí lo hicieron el 36\% optó por la opción de Letras, el $21 \%$ por Letras mixtas y el $29 \%$ escogió la opción de Ciencias. Respecto a la nota media obtenida en los estudios de secundaria resaltar que el $46 \%$ de la muestra obtuvieron una nota media de Aprobado, el $31 \%$ de Notable y un 23\% obtuvo un Bien.

Uno de los principales escollos a salvar en el entorno integrado, señalado por este grupo de estudiantes ha sido la dificultad con la asignatura de matemáticas. El inconveniente viene marcado por la complejidad en la adaptación de la nomenclatura al sistema braille, ya que la cantidad de símbolos que se originan es grande y se produce por la combinación de los seis puntos de la matriz generadora, representándose, además, en el papel de forma secuencial, lo que dificulta su lectura y memorización. Hay que tener en cuenta que el sistema braille se lee de forma analítica y se representa de la misma manera. Los conceptos matemáticos no tienen porque suponer una dificultad, pero sí la supone su lectura y escritura.

Las matemáticas son una de las asignaturas que menos agrada a los estudiantes de secundaria, si a esto unimos las dificultades de representación que suponen en sistema braille, podemos encontrar la explicación de la dificultad que tiene, a la hora de cursar asignaturas que impliquen cálculos matemáticos, para los alumnos con discapacidad visual y el abandono de opciones en las que este tipo de materias tienen un mayor peso. Nos aventuramos a apostar, en este sentido, más por la resolución de un problema metodológico al que todavía no se ha encontrado solución, que por una limitación impuesta por el déficit; 
aunque sería necesario un estudio en mayor profundidad en esta línea para poder dar resultados concretos.

Este condicionante, se repite cuando los alumnos llegan a la Universidad, de modo que aquellas personas que cursan alguna asignatura en la que se trabaja interpretando gráficos, por ejemplo economía, o asignaturas que conllevan la utilización de conceptos matemáticos, como estadística, tienen dificultades para aprobarlas. Corroborando esto, los alumnos ciegos entrevistados exponen que después de su experiencia, recomendarían a otros alumnos que a la hora de realizar estudios universitarios, elijan carreras en cuyos planes de estudio no se reflejen asignaturas con matemáticas.

\section{C) Factores Condicionantes de la Elección Vocacional}

En lo que se refiere a los factores que influyen en la toma de decisiones a la hora de realizar estudios universitarios, encontramos que las preferencias, recogidas en la respuesta «siempre me ha gustado», fue señalado por el $43 \%$ de los entrevistados como uno de los factores que les influyó en la decisión tomada. De la misma manera, el 80\% recomendaría a otros compañeros que la elección de los estudios se produjese en función de las preferencias personales: «Que elija aquello que más le guste, dificultades te encuentras siempre, pero hay que irlas superando y no pararse». La necesidad de contar con modelos, es una de las propuestas que también es apuntada por los estudiantes ya que el 33,3\% considera que la experiencia de otras personas con el mismo problema puede ayudar. Se indicó también, por el 33,3\%, la necesidad de tener en cuenta las salidas profesionales para personas con discapacidad. Si analizamos la respuesta indirecta que se da a esta cuestión en otros apartados del cuestionario, obtenemos que se contestó claramente en la línea de tener en cuenta la limitación funcional a la hora de elegir o se verbalizó claramente la importancia de solicitar asesoramiento, o consejo, a algún estudiante o profesional con la misma deficiencia visual.

Tradicionalmente se ha considerado a las aptitudes como uno de los condicionantes importante, sino el más, en el desempeño profesional y en la elección de carrera. Así, junto con las preferencias, una de las razones más repetidas para elegir una carrera, ha sido la de creer que se tenían aptitudes para realizarla, opción que fue señalada por el $43 \%$. Derecho es una de la salida académica que se elige fundamentalmente por pensar que se tiene una aptitud especial para ella, principalmente la memoria. Este hecho no deja de ser un tópico, atribuido socialmente a las personas ciegas o con baja visión, que parece tener su correlato en la percepción de los afectados.

\section{D) Tipo de Elección Vocacional}

La no existencia de modelos, junto con el rechazo de las matemáticas puede explicar que, aunque existe un aumento de este colectivo en los estudios superiores, estos estudiantes se centren especialmente en carreras denominadas «tradicionales», aquellas en las que un mayor número de profesionales con déficit visual desarrollan su actividad. No podemos olvidar que el sistema educativo no posibilita el ensayo y conocimiento de profesiones no tradicionales, en las que existen muy pocos modelos a seguir. Hay que partir del supuesto de que «sólo interesa lo que se conoce». El interés, fundamentalmente, se aprende en inte- 
racción con el ambiente, siendo factible la intervención en este área. Por eso, en el caso de las personas con discapacidad se hace imprescindible analizarlas, ya que suelen barajar pocas opciones vocacionales, limitándose en exceso a aquellos campos y profesiones que son tradicionales para su déficit. Ahondando en esta premisa, encontramos que las personas entrevistadas, se concentran en unas pocas escuelas y facultades. Podemos decir que son los grupos vocacionales Humanístico, Psicopedagógico, Sociojurídico y Biosanitario los escogidos preferentemente por los estudiantes, concentrándose en las carreras de Derecho, Psicología, Geografía e Historia, Filología y Fisioterapia (ver Fig. 4). Estos datos no hacen más que reflejar lo que distintos estudios revisados señalan, las personas con discapacidad visual restringen sus opciones a las que tradicionalmente han venido desempeñando (Bosen-Milona, 1994; Martínez Henarejos, 1993).

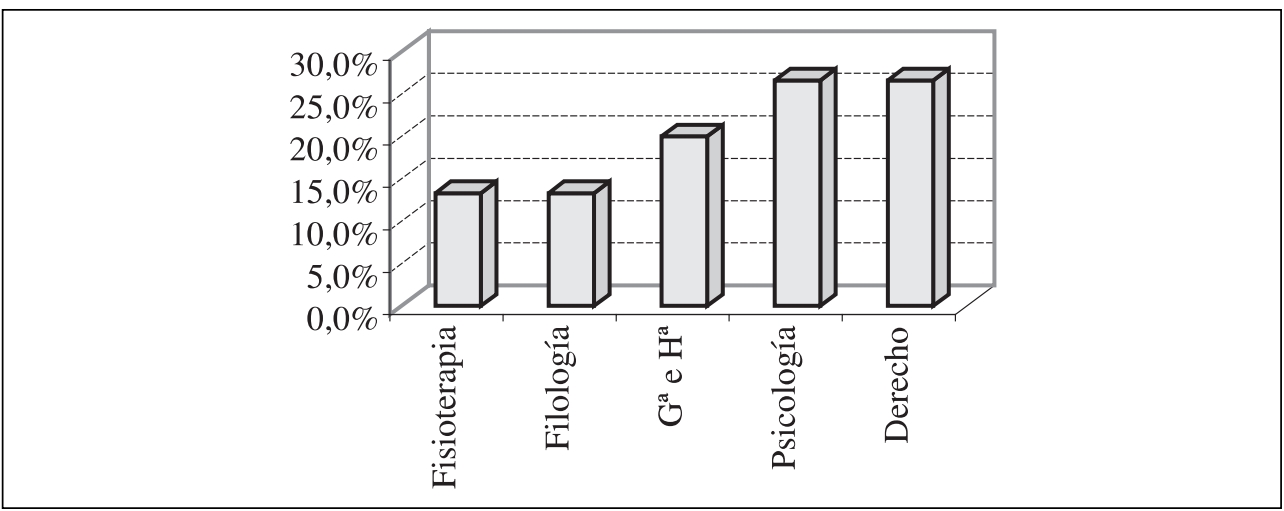

FIGURA 4.

Tipo de estudios que cursan los estudiantes entrevistados.

En esa tarea de modelado juega un papel muy importante la estructura laboral de la $O N C E$. Esta institución permite a personas afiliadas acceder a puestos de trabajo dentro de esta entidad, teniendo para ello preferencia frente a otros titulados. Además, hasta que el mercado esté saturado, los afiliados pueden trabajar en la venta del cupón. Esta situación, unida a la precariedad de empleo en las personas discapacitadas, está generando que socialmente se considere a éste un colectivo privilegiado, provocando, en algunos casos, ciertas actitudes de rechazo, sobre todo por parte de otros grupos de personas con discapacidad. Esto queda muy bien resumido en el comentario que al respecto realizó uno de los estudiantes entrevistados: «hemos pasado de ser pobrecitos ciegos a que se nos vea como nuevos ricos».

Tanto es así, que un alto porcentaje de estudiantes toman en consideración, entre sus expectativas profesionales, la posibilidad de trabajar para esta institución en el momento de terminar la carrera, y evidentemente esto puede condicionar su desarrollo de carrera; bien en trabajos que impliquen preparar oposiciones $(46,6 \%)$, bien recurriendo a la venta del cupón, de forma temporal, mientras encuentran un trabajo relacionado con sus estudios (40\%); e incluso una estudiante se plantea la posibilidad de realizar su desarrollo profesional como directivo en ella. Por otra parte, la posibilidad que esta entidad ofrece para poder 
trabajar durante el verano es un mecanismo, utilizado por el $26 \%$ de los estudiantes entrevistados, para financiar sus estudios.

Este tipo de asociaciones, que agrupan a colectivos con discapacidad, tienen un papel protector, como señala Miranda (1988). Si el individuo restringe sus contactos sociales a los ofrecidos por el colectivo de personas con discapacidad, sus posibilidades sociales pueden verse reducidas y por tanto verse perjudicado también su desarrollo vocacional. En el caso de los estudiantes entrevistados, hemos detectado que en mayor o menor medida, la mayoría de ellos realizan alguna actividad de las que oferta esta institución. Los viajes y salidas culturales, son las actividades en las que encontramos mayor participación de los entrevistados, en un $65 \%$ de los sujetos; el deporte adaptado, con un $40 \%$ de participación, estaría en segundo lugar; y la pertenencia al coro de esta institución, con un 13,3\% de participación; son en definitiva las más realizadas. Las personas que llevan a cabo la mayoría de sus actividades en entornos no integrados opinan que se encuentran más "cómodas» entre personas invidentes. Sin embargo hay que resaltar que estudios como el de Gottfredson (1986) señalan al aislamiento social junto con la limitación funcional, como factores de riesgo para un buen desarrollo de carrera de personas con discapacidad visual.

También la ONCE actúa como un recurso, inexistente para otros colectivos, que posibilita la obtención de becas para continuar estudios universitarios, y de adaptaciones técnicas que ayuden a la continuación de estudios e incluso en el puesto de trabajo a desempeñar.

La reacción social ante la propia discapacidad limita la conducta vocacional (Bagley, 1985). En esa respuesta social destaca el papel de las ideas preconcebidas, reales o no, en la percepción del mundo académico y laboral. Para conocerlas se formularon preguntas, abiertas y cerradas, referidas a ambas cuestiones. A continuación presentamos los resultamos más destacables.

\section{E) Percepción de Barreras Universitarias}

Con respecto a la Universidad, se formularon cuestiones referentes a barreras arquitectónicas y de infraestructura, de organización escolar, de material académico y de metodología utilizada en los centros de estudios.

Un grupo de problemas señalados por los estudiantes se refieren fundamentalmente a aspectos relacionados con la aptitud física, siguiendo la denominación de Alcantud (1994). Destacan, dentro de las barreras arquitectónicas y de infraestructura, la carencia de semáforos adaptados, que dificulta la movilidad entre edificios; la falta de adaptación de ascensores y paneles, la poca señalización de puertas y escalones o la mala iluminación dentro de los edificios.

En lo referente a la organización escolar la aglomeración y el exceso de número de horas de clase que imponen los nuevos planes de estudio son cargas que todo estudiante universitario soporta, pero en el caso de aquellos que padecen deficiencia visual estos problemas agravan el día a día en la Universidad.

La falta de adecuación del material académico, bien por la práctica, ampliamente extendida, de entregar material fotocopiado, que impide una buena lectura o la obtención de copias en soporte magnético o braille mediante su escaneado; bien por la falta de disposición de los libros de biblioteca para realizar transcripciones o grabaciones, son aspectos que uni- 
dos a la metodología empleada en la aulas con una utilización cada vez mayor de técnicas audiovisuales y la falta de adaptación de los sistemas utilizados para facilitar el acceso a los conceptos matemáticos, forman parte de esos aspectos que complican el paso de estos estudiantes por las aulas universitarias.

En otro orden de cosas la actitud de los compañeros es considerada por los estudiantes de la muestra buena, aunque también se señala que el desconocimiento por parte tanto de algunos alumnos como de profesores, unido a la actitud negativa mostrada por algún profesor, es lo que dificulta la realización de determinadas tareas. Un ejemplo de ello son las reticencias mostradas por algunos docentes para que sus clases sean grabadas.

Después de las preguntas relativas a su toma de decisión ante la opción universitaria, al igual que Salomone y Paige (o.c.) lo hicieran en su trabajo, se les interrogó sobre el consejo que darían a un adolescente con discapacidad visual a la hora de elegir una carrera.

Todas las personas ciegas ponen especial énfasis en que: «Hay que ser consciente de la discapacidad a la hora de elegir una carrera, no elegir una carrera que suponga un esfuerzo sobrehumano». Por lo que el factor relativo a la limitación funcional, señalado por Gottfredson (o.c), también se consultó en nuestro estudio. Las opciones señaladas como insuperables son Arquitectura y Medicina. La necesidad de recibir asesoramiento también es subrayada por el 33,3\% de la muestra. En algunos universitarios hemos detectado que la falta de asesoramiento vocacional en el momento de tomar una decisión les llevó a elegir sobre la base de unos criterios que pudieron no ser los más oportunos. En este sentido creemos que dicho asesoramiento es fundamental.

\section{F) Percepción de Barreras Laborales}

Tal vez sean las percepciones que se tienen respecto a la inserción laboral las que contengan un componente más subjetivo que el mostrado en el entorno universitario. Salomone y Paige (1996) encuentran la actitud negativa de la gente hacia la discapacidad visual, el pobre concepto de uno mismo, la negativa de los empresarios a contratar, las limitaciones en el transporte y, una planificación profesional y preparación vocacional inadecuadas o de baja calidad, como los aspectos que pueden dificultar la inserción socio-laboral del colectivo de personas con discapacidad visual.

En cuanto a los aspectos referentes a la actitud ante la discapacidad visual por parte de los empresarios y la dificultad para encontrar trabajo una vez terminada la carrera, este grupo de estudiantes no cree que encontrará una actitud negativa, dando un 66,6\% de respuestas a las alternativas, «bastante desacuerdo» y «total desacuerdo» ante la afirmación: «cuando termine mi carrera encontraré una actitud negativa de los empresarios hacia mí, debido a mi discapacidad». Por el contrario afirman que su preparación y talante les hará merecedores de un puesto de trabajo si se les da una oportunidad. Esta opinión queda reflejada en las respuestas dadas a la afirmación: «cuando acabe la carrera nadie confiará en mis posibilidades», concentrándose un $73,3 \%$ de respuestas en la alternativa de «total desacuerdo».

La adaptación de los puestos de trabajo que se va a desempeñar no es vista tampoco como una barrera. Aunque el 26,6\% piensa que los trabajos que pueden ocupar no están adaptados a sus características, todos ellos apuntan que las posibilidades de adaptación con 
medios técnicos es posible, ya que en la actualidad la ONCE adapta los puestos de trabajo de aquellos afiliados con contratos laborales.

Por el contrario, parece constatarse que las dificultades para encontrar trabajo se van a deber a la situación del mercado laboral más que a la propia discapacidad, aunque no deja de reconocerse que ésta puede influir. El 53,3\% señala que: «cuando termine la carrera tendrá las mismas dificultades para encontrar trabajo que sus compañeros videntes», lo que nos hace suponer que no nos encontramos ante personas con un pobre autoconcepto.

Podemos concluir que los estudiantes entrevistados están más preocupados por las barreras que encuentran dentro de la Universidad que por las posibles barreras del mercado laboral. Piensan, así mismo, que la principal dificultad para encontrar trabajo es la situación del mercado laboral, más que la discapacidad que sufren.

\section{Conclusiones}

La información recabada aún siendo descriptiva y una primera aproximación al tema que debe ser ampliada en posteriores trabajos, nos permite señalar la necesidad de ampliar o introducir, en algunos casos, los servicios de asesoramiento vocacional y educativos en la enseñanza secundaria, no sólo por la petición expresa realizada por gran parte de los estudiantes participantes en este estudio, sino también por el análisis de los datos recogidos sobre el transcurso de sus propios desarrollos vocacionales.

Se hace preciso plantear programas de asesoramiento vocacionales que amplíen y no restrinjan en exceso las opciones vocacionales a las típicamente tradicionales escogidas por las personas con déficits visuales (incluidas, en nuestro país las derivadas de la Organización Nacional de Ciegos).

La elección de estas opciones vocacionales tradicionales, muchas veces está relacionada con la «huída o evitación» de aquellas profesiones en cuya formación de base se encuentran asignaturas como las matemáticas u otras similares (estadística, etc.).

Así pues, en las intervenciones relacionadas con el desarrollo vocacional de estudiantes con déficits visuales se debe fomentar:

- La necesidad de presentar modelos, a los que poder observar e imitar, que representen opciones no tradicionales para las personas con déficits visuales.

- Actuaciones que lleven a superar las limitaciones metodológicas que el estudio de las matemáticas hoy por hoy representa, de forma que se pueda acceder a este tipo de contenidos formativos sin la complejidad que hasta ahora conllevan.

- La puesta en marcha de programas de intervención que cambien la actitud negativa hacia conceptos matemáticos; programas similares a los que se vienen desarrollando con mujeres para favorecer su acceso a opciones más amplias sin necesidad de restringirlas a opciones vocacionales tradicionales del género femenino. (Gimeno y Rocabert, 1998).

Del mismo modo y dada la influencia e importancia que tiene la familia en el desarrollo vocacional de los adolescentes y jóvenes, unido a las actitudes negativas que muchos padres suelen tener hacia las expectativas de formación y laborales de sus hijos e hijas con deficiencias visuales, incluida la sobreprotección que algunos muestran; hace imprescindible 
la participación de la familia en programas de asesoramiento, para mejorar y eliminar esas actitudes nada favorecedoras de la toma de decisiones vocacionales racionales e independientes, que todo proceso de asesoramiento vocacional debe contemplar como objetivo último, se tengan o no deficiencias visuales.

Por otra parte es justo reconocer el carácter algo más complejo que el desarrollo vocacional de personas con deficiencias visuales puede tener. Ello es debido, no tanto a la propia deficiencia, como a una serie de barreras u obstáculos que no suelen ser percibidos o padecidos por personas sin esos déficits. De nuestro estudio se desprende que en el contexto de la Universitat de València (Estudi General), y sin que se pueda generalizar sin más a otras universidades españolas, se hace preciso realizar actuaciones encaminadas a la detección y eliminación de barreras arquitectónicas, de infraestructura, la modificación de equipamiento, de la metodología utilizada en las aulas y de los materiales de estudio; posibilitando el acceso a la información escrita de estos estudiantes, y ajustando o modificando los sistemas de evaluación.

La institución debe proporcionar tanto la adaptación física como administrativa. En este sentido, en la Universitat de València (Estudi General) se lleva a cabo el trabajo de asesoramiento a este grupo de estudiantes por parte de la «Asesoría de Atención al Estudiante con Discapacidad». Para más información sobre el acceso de estudiantes con discapacidad a la universidad, iniciativas llevadas a cabo y principales vías de actuación, invitamos al lector a revisar el libro de Alcantud y otros (1999).

Por último resaltar que tal vez las barreras más importantes son aquellas menos explicitadas por los alumnos, pero que podemos inferir de sus respuestas. Nos parece fundamental la necesidad de incidir en el asesoramiento vocacional en períodos educativos anteriores, de manera que éste ayude a la toma de decisiones, y en el seguimiento de dicho asesoramiento en el período universitario. A su vez, creemos que existe un claro desconocimiento del mundo del trabajo, que se refleja en la no percepción de barreras para su inserción en él. Un acercamiento al desarrollo profesional a través de prácticas o convenios con empresas podría ser el mecanismo mediante el cual el estudiante eliminara percepciones erróneas respecto al mismo y así prevenir posibles problemas futuros.

\section{Bibliografía}

Alcantud, F. (1994). Analysis of the needs for the integration of disabled people in college. FEDORA Newsletter, January.

Alcantud, F. (1995). Estudiantes con discapacidades integrados en los estudios universitarios: notas para su orientación. En F. Rivas (Ed.), Manual de asesoramiento y orientación vocacional. Madrid: Síntesis.

Alcantud, F.; Ávila, V.; Asensi, M.C. (1999). La integración de estudiantes con discapacidad en los estudios superiores. Valencia: Universitat de València.

Ávila, V. (1999). Análisis de la problemática de los alumnos con deficiencias visuales en los estudios universitarios: El caso de la Universitat de València (Estudi General). Valencia: Tesis de Licenciatura. Universitat de València (E.G).

Bagley, M. (1985). Service providers' assessment of the career development needs of blind and visually impaired students and rehabilitation clients and the resources available to meet those needs. Journal of Visual Impairment and Blindness, 79 pp. 434-443. 
Bosen-Milona, R. (1994). Le défi de l'éducation spécialisée. Le Valentin Haüy, n. 35, pp. 7-9.

Bush-Lafrance, B. (1988). Unseen expectations of blind younth: Educational and occupational ideas. Journal of Visual Impairment and Blindness, 82, 132-136.

Denzin, N. K y Lincolns, Y.S. (1994). Handbook of Qualitative Research. California: Sage Publications. Thousand Oaks.

Fitzgeral, L.F., y Crites, J.O. (1980). Toward a career psychology of women: what do we know? What do we need to know? Journal of CounselingPpsychoogy, 27, pp. 44-62.

Fundación Once (1996). Minusvalía e inserción laboral en la Comunidad Valenciana. Madrid: Escuela Libre Editorial.

Gimeno, M.J. y Rocabert, E. (1998). Barreras percibidas por las mujeres en su proceso de toma de decisiones vocacional. Revista de Orientación y Psicopedagogía. Vol. 9, n. 15, $1^{\text {er }}$ Semestre.

Gottfredson, L. (1986). Special groups and the beneficial use of vocational interest inventories. En Walsh y Osipow: Advances in vocational Psychology.

Hurst, A. (1993). Steps towards graduation: Acces to higher education and people with disabilities. London: Avebury Press.

Hutto, M. (1993). Family influence on the career advancement of legally blind female college fraduates. Tesis doctoral. Mississippi State University.

Martínez Henarejos, A. (1993). Integración laboral de las personas con ceguera o deficiencia visual: actuación de la ONCE. III Jornadas de Psicología de la Intervención Social. Madrid: Instituto Nacional de Servicios Sociales. Pp. 941-951.

Miranda, M.J. (1988). Actitudes ante la deficiencia visual y la ceguera, en relación con la formación profesional y el empleo, de dos grupos sociales: los empleadores actuales y potenciales y los propios deficientes visuales y ciegos. Madrid: Proyecto de investigación financiado por la ONCE.

Rabby, R. y Croft, D. (1989). Take charge: A strategic guide for blind job seekers. Boston: National Braille Press.

Rivas, F. (Ed.) (1995). Manual de asesoramiento y orientación vocacional. Madrid: Ed. Síntesis.

Rocabert, E. (1999). Problemática y desarrollo vocacional de los estudiantes con minusvalías sensoriales y motóricas. En F., Rivas y M. L., López, (Ed.) Asesoramiento vocacional de estudiantes con minusvalias fissicas y sensoriales. Servicio de publicaciones. Valencia: Universitat de Valéncia (E.G).

Rosa, A y Ochaita, E. (Comp.) (1993). Psicología de la ceguera. Madrid: Alianza Editorial.

Salomone, P.R. y Paige, R.E. (1996). Employment problems and solutions: Perceptions of blind and visually impaired adults. Vocational Guidance Quarterly, 33, 147-156.

Fecha de recepción: $25-I V-00$

Fecha de revisión: 18-IV-01

Fecha de aceptación: 20-V-01 


\section{ANEXO I: CUESTIONARIO GUÍA}

\begin{tabular}{|c|c|}
\hline int & $\begin{array}{c}\text { CUESTIONARIO SOBRE NECESIDADES DE } \\
\text { LOS UNIVERSITARIOS CON } \\
\text { DISCAPACIDAD VISUAL }\end{array}$ \\
\hline
\end{tabular}

\section{DATOS DE IDENTIFICACIÓN}

1. Edad:

2. Sexo:

3. Tipo de discapacidad:

- Ciego total

$\square$ Deficiente visual

$\square$ Degenerativa

$\square$ Resto visual...........

$\square$ Ceguera nocturna

4. Grado de discapacidad, según certificado de minusvalía:

5. Momento de aparición:

- No veía cuando nací.

Entre los 0 y 3 años.

Entre los 4 y 6 años.

Entre los 7 y 12 años.

- Entre los 13 y 18 años.

- A partir de los 19 años.
La enfermedad la tenía al nacer pero me la detectaron:

Entre los 0 y 3 años.

Entre los 4 y 6 años.

Entre los 7 y 12 años.

E Entre los 13 y 18 años.

口 A partir de los 19 años.

6. Causa de la discapacidad:

\section{DATOS FAMILIARES}

7. ¿Hay alguna otra persona ciega o DV en tu familia?:

$\square$ Mi padre

口 Mi madre

Alguno de mis hermanos.

8. Ocupación de los Padres.

Obrero especializado

Asalariado no especializado

Empresario

Amo/a de casa

Profesional liberal

Docente
口 Algún pariente cercano (tíos, primos...)

Algún pariente lejano

$\square$ Ninguna.
Ocupación del Padre

1

2

3

4

5

6
Ocupación de la Madre

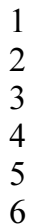

6 
Desempleado

Trabajador autónomo

Jubilado

Fallecido
7

8

9

10
7

8

9

10

9. Profesión de los Padres.

Padre:

Madre:

10. Nivel de estudios de los padres.

Sin estudios

Primarios incompletos

Primarios completos

Bachillerato

Diplomado Universitario

Licenciado Universitario

Doctor

Estudios del padre

1

2

3

4

5

6

7
Estudios de la madre

1
2
3
4
5
6
7

\section{DATOS ACADÉMICOS}

11. Facultad o Escuela:

Universidad:

12. Curso: Año en que iniciaste tus estudios:

13. Forma de acceder a la Universidad

$\mathrm{COU}$ FP

Mayores 25 años

Segunda carrera

14. Momento en que iniciaste la escolarización:

口 Antes de los 4 años

Entre 4 y 6 años

Después de los 6 años

15. ¿Dónde realizaste tus estudios anteriores?

Centro Público

C. Privado

Estudios Primarios

C. Concertado

C. Específico para Ciegos o DV

1
2
3
4

BUP ó FP
1
2
3
4

COU
1
2
3
4

16. Nota media enseñanza secundaria (BUP/COU o FP):

17. Opción de BUP/COU o especialidad realizada en FP: 
18. ¿Qué asignaturas preferías en los estudios medios?:

$\square$ Matemáticas
$\square$ Física
$\square$ Literatura
$\square$ Historia
$\square$ Idiomas

Otros:

$\square$ Dibujo
Clases prácticas y tecnología
Biología
Química
$\square$ Deportes

19. Cómo financias o has financiado tus estudios?:

Te los paga tu familia.

Consigues beca de Estudios

$\square$ Consigues beca de la ONCE

Otros:
$\square$ Trabajas
口 Trabajas durante el verano

20. ¿Cuántas horas estudias o estudiabas normalmente durante tu estancia en la Universidad?

Menos de dos horas diarias. Entre 3 y 4 horas al día.

$\square$ Entre 2 y 3 horas al día.

Más de 4 horas al día.

\section{FACTORES QUE INFLUYEN EN LA CONDUCTA VOCACIONAL:}

21. ¿Quien ha influido o influyó en tu decisión de estudiar?

$\square$ Tu familia

$\square$ Tus amigos

Algún profesor/asesor

Otros:
- Algún miembro de la ONCE

Nadie en especial.

22. Ante la decisión de continuar estudios universitarios, tu familia te mostró:

$\square$ Apoyo.

$\square$ Oposición. ¿por qué motivo?:

$\square$ Indiferencia.

23. Durante tus estudios universitarios, tu familia te muestra o mostró:

a Apoyo.

Oposición. ¿por qué motivo?:

$\square$ Indiferencia.

24. Cuando tienes que realizar algún trámite buscas:

Que te acompañe alguien de tu familia.

$\square$ Que te acompañe algún amigo.

$\square$ Prefieres hacerlo tú sólo/a.

Otros: 
25. ¿Qué haces en tu tiempo libre?

Cantar en un coro

Tocar algún instrumento ¿cual?:

Practicar deporte ¿cual?:

Salir con amigos de copas

Ir al cine con amigos

$\square$ Oír música en casa

Leer literatura

Dar largos paseos

El tiempo que tienes libre lo dedicas a estudiar

Otros:

26. ¿Cómo acudes normalmente a la facultad/escuela o puesto de trabajo?:

Con perro guía.

- Sólo

Alguien te acompaña.

27. Cuando necesitas conocer un nuevo itinerario:

- Solicitas ayuda al TRB de la ONCE.

Tu familia/amigos/profesor se encargan de ayudarte.

$\square$ No cambias de itinerarios.

28. Ante un desplazamiento imprevisto (p. ej. tienes que acudir a una cita en un lugar no conocido y nadie ha pasado a recogerte)

$\square$ Llamas por teléfono a tu familia o a algún amigo para que pase a por ti.

$\square$ Te aventuras a salir a la calle y pides ayuda.

$\square$ Llamas a un TRB de la ONCE para que te ayude.

$\square$ Nunca te puede ocurrir algo así.

$\square$ No acudes a la cita.

\section{FACTORES QUE INFLUYEN EN LA ELECCIÓN VOCACIONAL}

29. La carrera en la que te encuentras, o que has realizado, es la que querías estudiar:

$\square$ SI

$\square \mathrm{NO}$

30. Si tu respuesta es NO, ¿qué carrera te hubiera gustado realizar?:

31. Señala las causas por las que NO realizaste la carrera que deseabas.

$\square$ Mis aptitudes.

La nota media que se pedía para entrar.

- Las posibilidades económicas de mi familia.

$\square$ La dificultad y duración de los estudios

$\square$ Por no encontrarse cerca de mi residencia.

- La falta de salidas profesionales 
Por oposición familiar

$\square$ Por ser desaconsejada por el asesor.

$\square$ Por que no conozco a ninguna persona con deficiencia visual que la haya realizado.

Otros:

32. ¿Por qué escogiste la carrera que cursas o que cursaste?

$\square$ Porque les gustaba a mis padres

- Porque me lo indicaron en la ONCE

$\square$ Por influencia de algún profesor/asesor.

- Porque puedo compaginarla con mi trabajo o hobby...

$\square$ Porque creo que tengo aptitudes para ello.

- Por ser la más adecuada tras un proceso de asesoramiento.

$\square$ Porque siempre me ha gustado.

$\square$ Por tradición familiar.

D Para aumentar mi cultura general.

Porqué mis amigos la habían elegido.

$\square$ Porque conocía algún deficiente visual que la había realizado o la estaba realizando.

- Por las salidas profesionales que tenía.

Era la qué menos problemas me planteaba para llegar al centro de estudios. Otros:

33. Qué te planteas hacer cuando termines tu carrera o qué hiciste cuando terminante tu carrera:

Opositar, ¿a qué y dónde?:

- Sólo estudio o estudié para ampliar mi cultura, no espero dedicarme a ello de manera profesional.

Montar mi propia empresa o negocio. ¿Cuál?:

D Buscar trabajo en la ONCE

Buscar trabajo en empresas privadas. ¿Ámbito o sector?:

Quiero conseguir ser docente e investigador en la Universidad.

- Continuar mi formación a través de masters y cursos de especialización.

$\square$ Realizar otra carrera.

Otros:

34. ¿Qué aconsejarías a un adolescente con deficiencia visual o ciego a la hora de elegir una carrera?

Qué elija aquello que más le gusta, lo va a tener difícil en cualquier lugar.

$\square$ Que realice estudios dentro de la ONCE (p. Ej. fisioterapia)

$\square$ Qué hable con personas con déficit visual que están realizando estudios universitarios o que hayan acabado.

$\square$ Que tenga en cuenta las posibles salidas profesionales para una persona con discapacidad visual.

$\square$ Que pida asesoramiento en la ONCE.

$\square$ Que pida asesoramiento en el instituto. 
Que no se complique la vida y no siga el camino universitario Otros:

\section{BARRERAS UNIVERSITARIAS Y LABORALES}

\section{Señala tu grado de acuerdo con las siguientes afirmaciones:}

\section{Teniendo en cuenta que:}

TA Totalmente de acuerdo

BA Bastante de acuerdo

BD Bastante en desacuerdo

TD Totalmente en desacuerdo

1. Cuando termine mi carrera voy a tener más dificultades para encontrar trabajo que mis compañeros videntes.

TA BA BD TD

2. Cuando termine mi carrera encontraré una actitud negativa de los empresarios hacia mi, debido a mi discapacidad.

TA BA BD TD

3. La dificultad de los estudios unido a mi discapacidad pueden limitar las posibilidades de realizar estudios universitarios.

TA BA BD TD

4. Mis capacidades para realizar estudios universitarios están limitadas por mi discapacidad.

TA BA BD TD

5. Las características del desplazamiento (tipo de transporte, situación del centro...) limitan mis posibilidades de realizar estudios universitarios.

TA BA BD TD

6. Creo que los puestos de trabajo que puedo ocupar, en la actualidad, no están adaptados a mis características.

TA BA BD TD

7. Soy extrovertido/a y no me cuesta relacionarme con la gente.

TA BA BD TD

8. Me gusta asistir a fiestas y reuniones de amigos.

TA BA BD TD

9. Tengo muchos amigos.

TA BA BD TD

10. No acabaré la carrera, tengo demasiadas limitaciones para hacerlo.

TA BA BD TD

11. Cuando acabe la carrera, nadie confiará en mis posibilidades.

TA BA BD TD

12. En mi facultad o escuela he encontrado los mismos problemas que el resto de mis compañeros.

TA BA BD TD

13. En la universidad he encontrado gran desconocimiento sobre personas con DV por parte de los profesores.

TA BA BD TD

14. En el campus he encontrado muchas barreras arquitectónicas.

TA BA BD TD

15. En las clases se utilizan mucho las técnicas audiovisuales, que dificultan mi seguimiento de las clases.

TA BA BD TD

16. La metodología (forma de dar la clase) que se emplea en mi facultad/escuela se ha adaptado a mis características.

TA BA BD TD 
17. En la universidad no he tenido material adaptado a mis características.

TA BA BD TD

18. Durante los exámenes no tengo suficiente tiempo para responderlos.

TA BA BD TD

19. Los plazos para disponer de los libros de biblioteca son cortos para mi.

TA BA BD TD

20. Necesito estar en primeras filas, durante las horas de clase, y nunca encuentro sitio.

TA BA BD TD

21. La infraestructura de mi facultad es una gran barrera para mi (paneles mal iluminados, ...)

TA BA BD TD

22. A la hora de hacer el examen negocio, con la mayoría de los profesores, la forma de hacerlo.

TA BA BD TD

23. La mayoría de profesores antes de hacerme un examen me preguntan como quiero hacerlo.

TA BA BD TD

24. La mayoría de profesores han adaptado el examen, según lo que yo les he sugerido.

TA BA BD TD

36. Escribe las tres dificultades más importantes que has encontrado en la universidad: 